\title{
PLANNING TRIPOLI METRO NETWORK BY THE USE OF REMOTE SENSING IMAGERY
}

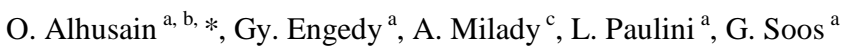 \\ ${ }^{a}$ UVATERV Engineering Consultants C.C. Ltd., H-1537 Budapest 114, P.O. Box 453/421, Hungary, \\ engedy@uvaterv.hu,paulini@uvaterv.hu,soos@uvaterv. \\ ${ }^{\mathrm{b}}$ Budapest University of Technology and Economics, H-1111 Budapest, Muegyetem rkp. 3, Hungary, \\ alhusain@eik.bme.hu \\ ${ }^{\mathrm{c}}$ Railroad Project Execution and Management Board, P.O. Box 82376 Swani Road, Tripoli, Libya \\ railwayslibya@lttnet.net
}

KEY WORDS: Urban, Planning, City, Imagery, Aerial, Satellite, Multitemporal

\begin{abstract}
:
Tripoli, the capital city of Libya is going through significant and integrated development process, this development is expected to continue in the next few decades. The Libyan authorities have put it as their goal to develop Tripoli to an important metropolis in North Africa. To achieve this goal, they identified goals for the city's future development in all human, economic, cultural, touristic, and nonetheless infrastructure levels. On the infrastructure development level, among other things, they have identified the development of public transportation as one of the important development priorities.

At present, public transportation in Tripoli is carried out by a limited capacity bus network alongside of individual transportation. However, movement in the city is characterized mainly by individual transportation with all its disadvantages such as traffic jams, significant air pollution with both carbon monoxide and dust, and lack of parking space.

The Libyan authorities wisely opted for an efficient, modern, and environment friendly solution for public transportation, this was to plan a complex Metro Network as the backbone of public transportation in the city, and to develop and integrate the bus network and other means of transportation to be in harmony with the planned Metro network. The Metro network is planned to provide convenient connections to Tripoli International Airport and to the planned Railway station. They plan to build a system of Park and Ride $(\mathrm{P}+\mathrm{R})$ facilities at suitable locations along the Metro lines.

This paper will present in details the planned Metro Network, some of the applied technological solutions, the importance of applying remote sensing and GIS technologies in different planning phases, and problems and benefits associated with the use of multi-temporal-, multi-format spatial data in the whole network planning phase.
\end{abstract}

\section{INTRODUCTION}

Planning of the Tripoli Metro Network was carried out for the first time in the period of 1984-1990. However, the revision of that original plan was done from scratch producing almost new plan. The new plan presented into this study took into consideration the city topography, its future development plans, population growth figures both in numbers and in distribution, and trends of age and occupations, future transportation demands were estimated until the year 2025. Accordingly Tripoli Metro Network was proposed to be consisting of three lines A (green), B (red), and C (blue) crossing the city from east to west and from north to south with good coverage of the inner parts of the city, with a total of transportation length of $99.5 \mathrm{~km}$, and a total of 72 stations, the total length of the network including service sections will be $106 \mathrm{~km}$. Figure 1, shows the schematic representation of Tripoli Metro Network, while figure 2, shows the layout of the Network.

Beside the generic elements of the planning process, the revision study dealt with many areas related to the planning process. Computer aided design of the project can't be carried out efficiently without the use of digital format displaying as a substitution to the classical planning hardcopy base maps. Digital spatial data usually is casted into one of two formats, either vector or raster format. Taking into consideration that vector digital maps of the project site were unavailable, so planners of the project at UVATERV (acronym of the capital letters of Út VAsút TERVezés, Hungarian words meaning Road and Railway Planning) opted to carry out their studies and analysis on raster format digital maps (digital imagery).

Planning activities in the original planning study of the period 1984-1990 were conducted using hardcopy maps provided by the contracting Libyan Railroad Project Execution and Management Board (RPEMB). The provided maps were prepared by the Polish POLSERVICE-WADECO in the period of 1979-1981. Production of the maps depended on 1:15000 scale aerial photographs, where aerial photography was carried out in the same period of 1979-1981. The datum of the mapping system is the ELD79, the projection system was the Transverse Mercator's 7th zone. The Mediterranean Sea Mean Level was chosen as an elevation datum. The analogue aerial photographs were data captured to produce 1:1000 and 1:5000 scale map segments. Content of these map segments includes buildings, fences, roads, earth roads, paths, and field forms.

Legal and administrative borders and some lines of the surface infrastructure were shown on these map segments. In non-urban areas $2 \mathrm{~m}$ interval contours represented, on the other hand in urban areas tagged elevation points can be found. Map keys provide reference to land use, buildings, and vegetation.

\footnotetext{
* Corresponding author.
} 
Documentation found on the map is written in both Arabic and English languages, these are mainly street names, descriptions of area status, and construction. Many other related data have been registered on the maps such as the map sheet number, a mapping scale, and control network coordinate values. Non-theless, useful index of map grid and map symbols can be found on each map segment. Figure 3, shows an analogue map sheet of the city of Tripoli.

These maps of Tripoli, undoubtedly -at that time- have had surpassed in their technical quality and content all other maps prepared previously about the city. At the same time and since then, the city has gone through many large scale changes that made these good quality maps obsolete, rendering them to an unsuitable tool in the revision study of the Metro Network.

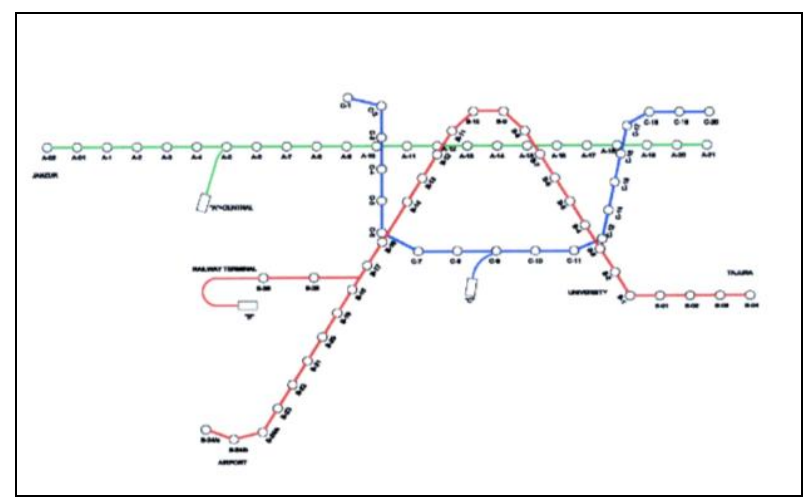

Figure 1. Schematic representation of Tripoli Metro Network

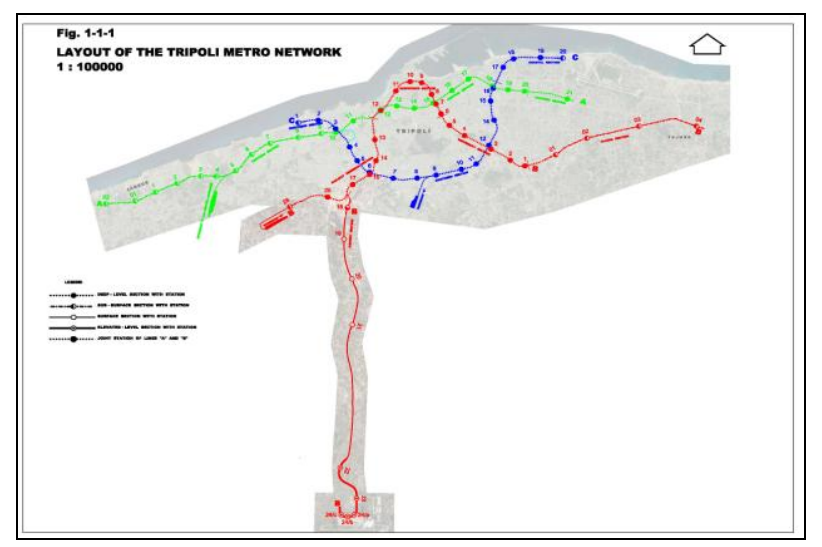

Figure 2. Planned layout of Tripoli Metro Network

Following practices of the time, many buildings and establishments were not shown on that map, a fact in itself devaluated the usability of the map for such a study. The technical basis of the map also became old and not very usable, knowing that the ELD79 datum severely limits the applications of GPS-based measurements. Nonetheless, although the map segments have been scanned and put in a coordinates system, which in theory transfer them to be used as a kind of computerbased raster map, the resulting product falls short of modern time standards and our expectations and requirements.

\section{BASE MAP OF THE TRAFFIC STUDY}

To carry out the Revision of the Metro Network Study, and especially to facilitate field work, there was a natural need to acquire an up-to-date mapping material. At the beginning of the revision work, it was unknown whether the contracting partner (RPEMB) will be able to provide the reviewing teams with such mapping material, and if so, what kind of quality; therefore the decision was made to look for a high quality and an up-to-date geographic data source where computer assisted design and modern technology can be efficiently applied. To satisfy the above mentioned criteria, the choice fallen on the CitySphere satellite image products of Tripoli. Figure 4, shows satellite mosaics covering the whole Metro planning area.

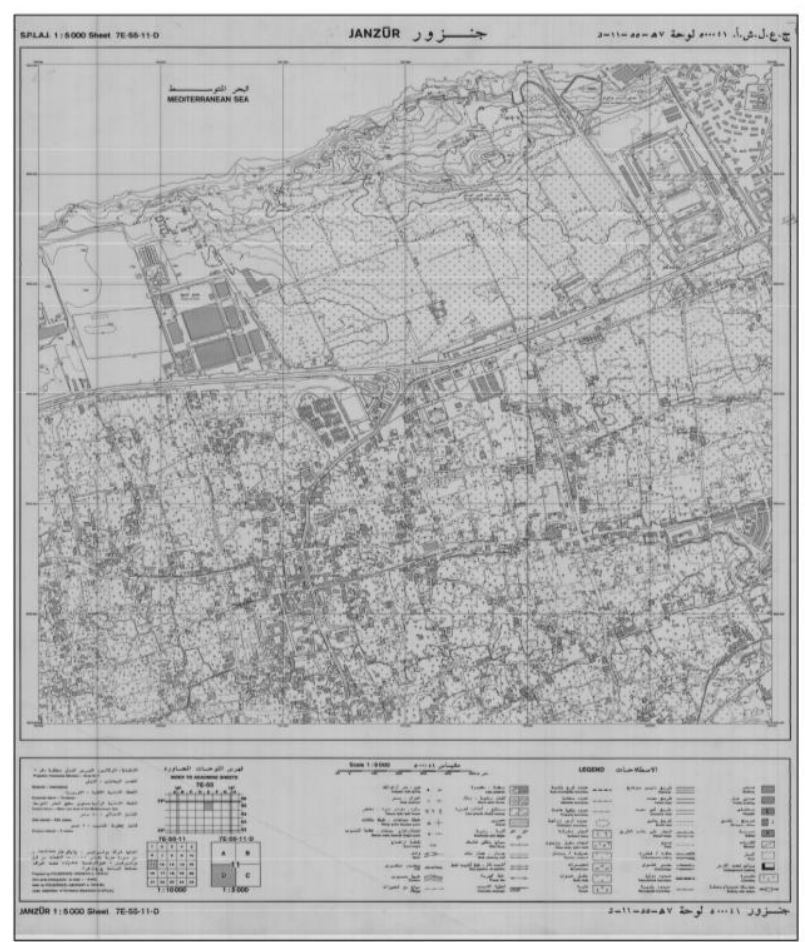

Figure 3. Analogue map sheet of Tripoli, Libya

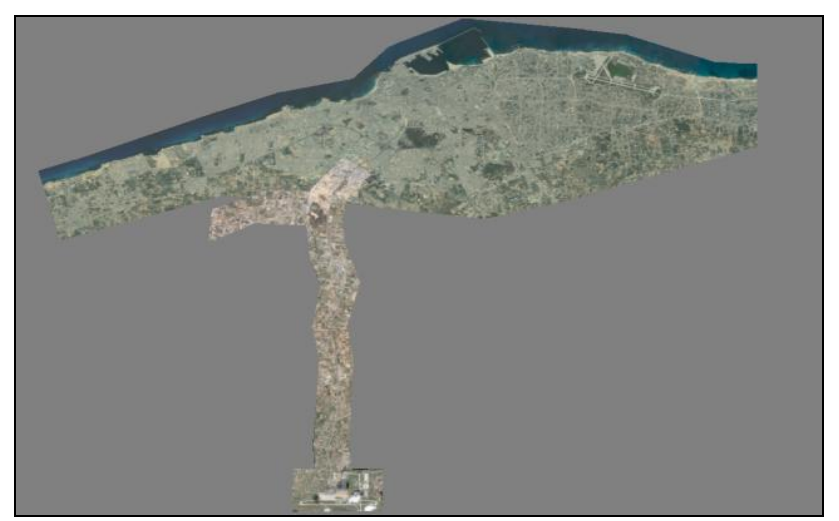

Figure 4. Satellite mosaics of Tripoli 
CitySphere images are high-resolution images acquired by QuickBird satellite; they were introduced by DigitalGlobe to provide surveyors, city planners, and geographic information systems specialists with a comprehensive, current, and repeatable image data covering major cities. These images provide the foundation and geographic context for a wide range of applications that require an up-to-date imagery; they are ideally suited for applications requiring consistent, reliable, and current data coverage of study sites where there is a need to integrate imagery into the workflows and decision-making processes.

CitySphere images used in this project are a collection of colour image mosaics acquired over Tripoli city in and around March 2006, these mosaics were arranged, co-registered, and projected resulting in an ortho photograph mosaics. The resolution of satellite imagery is $60 \mathrm{~cm}$, projected according to the WGS-84 datum system where the $33^{\text {rd }}$ zone of the Universal Transverse Mercator projection (UTM) was used. The individual image mosaics were aligned together to cover the whole area of Tripoli city, and put into GeoTiff image format which is very much suitable for computer aided design and geographic information systems related applications. Software used in processing these images included AutoCAD, GIS, and image processing programs.

To cover all areas where the Metro network is planned, the above mentioned image was complemented by a separate satellite image covering narrow path extending from the borders of the city to Tripoli International Airport and centered on the axis of the Airport highway which is aligned with the planned (B) Metro line. This last ordered image was acquired on March 2008 and has similar technical characteristics as the first one.

The satellite imagery has the advantage of providing coherent and comprehensive representation of earth's surface objects being imaged. Regarding their content, they truthfully reflect the facts on the ground at the time of acquiring the image (Lillesand and Kiefer, 1979; Detrekoi et al, 1996). However, in a city like Tripoli undergoing dynamic development and extremely rapid changes, the facts on the ground change fast too. Another important reason supporting the choice of satellite imagery as a base map is that such images can easily be modified to suite the computer assisted planning of the project, this is achieved with an acceptable relative accuracy (Alhusain, 2007).

Unfortunately, the nominal $2.5 \mathrm{~m}$ horizontal accuracy provided by the distributor of the images didn't prove to be true in all cases, especially in real life applications. This was especially critical issue when fitting and harmonizing some technical aspects of this project to other similar significant development projects executed in Tripoli. Coordinates transformations carried out on imagery didn't bring satisfactory results, for this reason a process of co-registration by identifying identical points on different multi-temporal images and carrying out local image transformations became necessary. Further significant problem was that the satellite imagery did not contain altitude information.

Overall, after carrying out the necessary geometric corrections, transformations, and co-registration the ordered satellite imagery has satisfied the required accuracy needs. By producing the necessary orthomaps it was possible to greatly facilitate the field work of the Metro planning teams and to support the inoffice work of Metro lines design, and imposition of Metro stations in their suitable locations. Nonetheless, the acquired images proved to be cost effective solution during the course of establishing the concept of the traffic study and the decision making process of the Metro network. At the same time satellite imagery constitutes correct and impressive technical basis for documentation and presentation of plans and technical solutions prepared through the traffic study (Alhusain, 2007; Engedy 2008).

\section{MAPS USED IN THE PLANNING STUDY REVISION}

During field visits, and data collection activities of January 2008. Our company and its teams got in contact with other experts responsible for planning and execution of similar big development projects in Tripoli. One of the important meetings took place between our teams and the Swedish subcontractor of the National Consulting Bureau of Libya (NCB). The NCB and its Swedish subcontractor are responsible for the city's future urban development; specifically they are working on the "Third Generation Planning Project" which envisages the urban development of the city until the year 2025. The managers of this project had indicated that their task, besides preparing the development plans for the city and its surrounding region, was to create totally new and modern technological principals dependent base map. This base map relies on $10 \mathrm{~cm}$ resolution aerial photographs acquired over Tripoli in 2008. Expert-level meetings between our teams and the NCB's have shown that the finished parts of the base map also constitute a useful tool capable of supporting the demands dictated by mapping tasks of the Revision of the Metro Planning Study. Steps were taken by our contracting partner (RPEMB) to provide us with these aerial photographs, the efforts of our partner were successful, and we received the aerial photographs in August 2008, figure 5 , shows an example of these photographs. Aerial photographs serving as the basis for producing the base map were acquired over Tripoli in autumn of 2006 and the beginnings of 2007 . Flight height was $1000 \mathrm{~m}$, out of the raw aerial photographs ortho photographs of 10 and $30 \mathrm{~cm} /$ pixel resolution were produced. Photogrammetric data capturing of the aerial photographs to produce vector maps has begun at the (NCB).

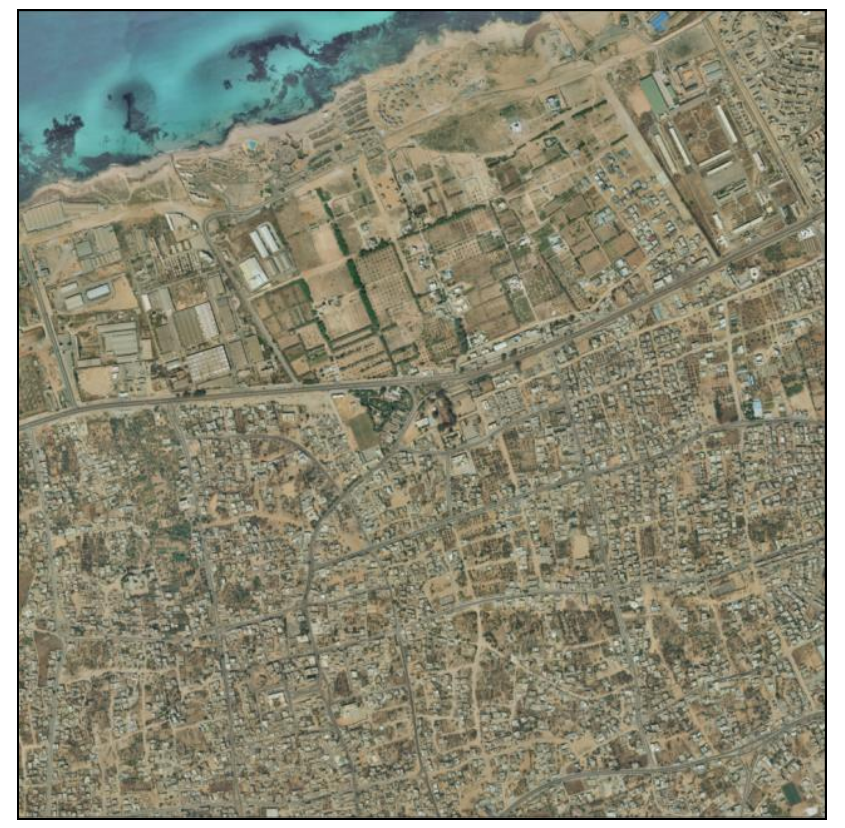

Figure 5. Aerial photograph covering one of the Metro planning areas

The (NCB) provided our experts a $30 \mathrm{~cm} /$ pixel resolution ortho photograph mosaics. According to their information, these mosaics in their present form are not considered to be a final 
product; rather they are considered a temporary work material, because in the implemented digital terrain model, instead of the Libyan Mean Sea Level altitude datum, they used the EGM96 geod related altitude data. This is according to the (NCB) estimation results in 40-60 $\mathrm{cm}$ mean error, which they plan to compensate for in the final ortho photograph products. The map was prepared on the $7^{\text {th }}$ zone of the Libyan Transverse Mercator Projection, which was tied to the LGD2006 datum. With this solution, using GPS-based measurements becomes possible without limits, taking into consideration that conversion between the WGS-84 and the LGD2006 datums can be easily solved. Coefficients needed to carry out the 3-parameters datum transformation are provided by the Surveying Department of Libya. In doing so, harmonization among development projects going on simultaneously in the city becomes possible. Further convenience service is reached in which GeoTiff format becomes available by cropping the ortho photographs along the edges of the Transverse Mercator projection mosaics. The mapping materials provided by the NCB were masked in many places for security reasons. Taking into consideration that the Metro network criss-crosses the inner city and intersects with some areas missing coverage in the NCB's aerial photographs, we had to substitute the missing parts with cuts from the satellite imagery, then apply elastic sheet transformation on these image cuts, and then they were co registered on aerial photographs. A further significant problem experienced is that the ortho photograph maps do not provide elevation data for the Metro network planning process. As a solution, we used elevation data from the above mentioned hard copy map scanned as a raster data. During transformation we took into consideration the effects of the differences of the datums of the photographs, and then the contours and the photograph of the discrete altitude points were put in vector forms. This practically means that plans prepared during the revision of the plan study of Tripoli Metro are relative to base surface determined by medium elevation of the Mediterranean Sea.

Overall, it can be said that the produced final map served well the accuracy requirements of $\pm 1 \mathrm{~m}$ in both latitude and altitude levels (horizontal and vertical directions) of the revision study of planning the Metro network. However, it should be noted that this product is not suitable for further detailed execution phases and tasks, for example it is not suitable for an execution plan. Execution plan, especially in densely built-up urban environments, data accuracy in the range of centimeter is needed. In essence such accuracy can be achieved only by terrestrial geodetic survey (Engedy, 2008).

\section{PLANNING THE METRO LINES}

From line designer's point of view, it is necessary to have a whole detailed picture of the planning area. In Tripoli Metro case the choice was to use a detailed base map which was casted in a well-known and technically sound projection system that covers the whole planning area. The projection choice of a base map that was not prepared by classical terrestrial geodesy happened for three main reasons: First, the planning area was so large that surveying it by terrestrial means was not an option. Second, at the beginning of the planning process, there was no final decision about the exact line alignments, and consensus were undergoing among different Libyan authorities about the exact alignment locations, this fact excluded the possibility for the designers to consider only narrow stripes of land for the purpose of lines alignments. Third, the time frame allocated for the revision study excluded the possibility of following lengthy terrestrial surveying works.
The availability of satellite imagery and aerial photographs during different stages of the planning process has made it possible to flexibly change line alignments according to the continuous negotiations and agreements between the contracting Libyan (RPEMB) and other Libyan authorities. Figure 6, shows deep alignment line that is led away from a planned traffic node. Figure 7, shows subsurface alignment line planned not affecting buildings and leaving half the street open during construction. Figure 8, shows that during construction only half of the highway needs to be closed. Figure 9, shows Metro line aligned avoiding a traffic node. And figure 10, shows Metro line aligned avoiding a bridge foundations.

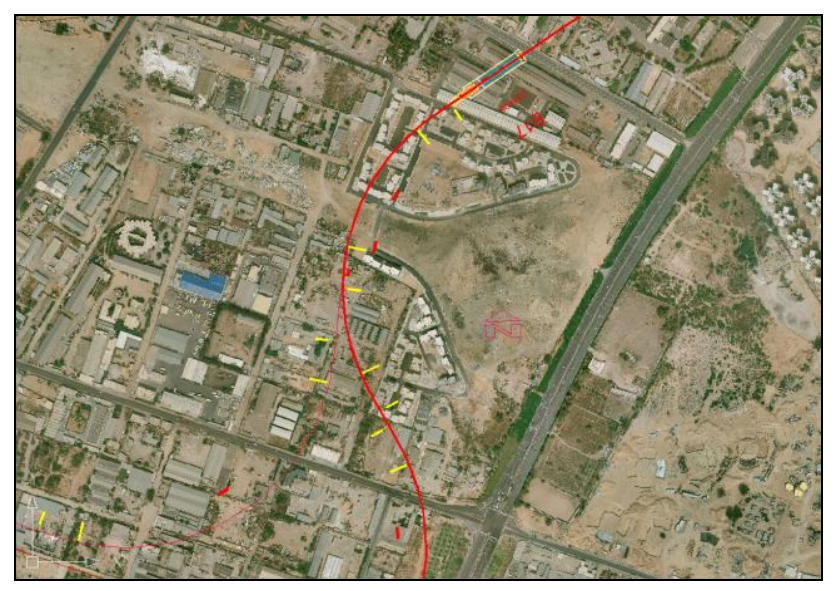

Figure 6. Deep alignment line led away from a planned traffic node

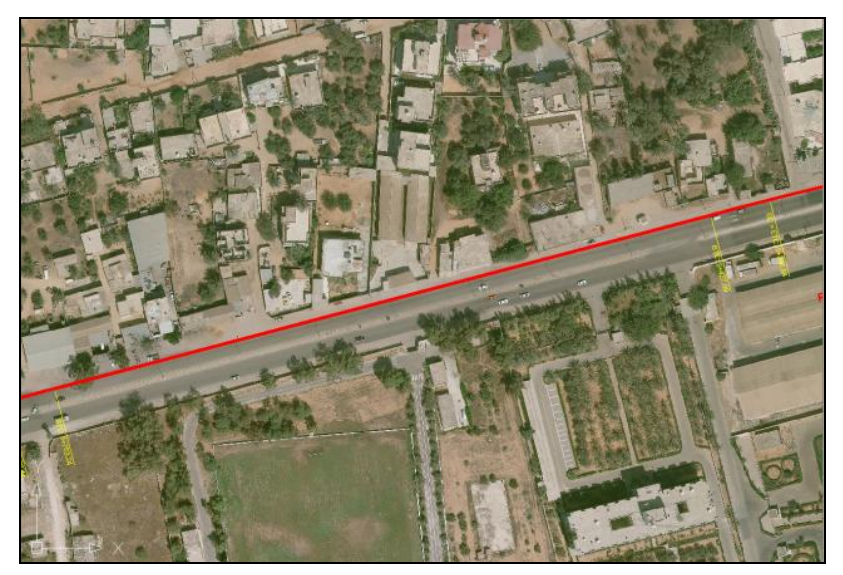

Figure 7. Subsurface alignment line not affecting buildings 


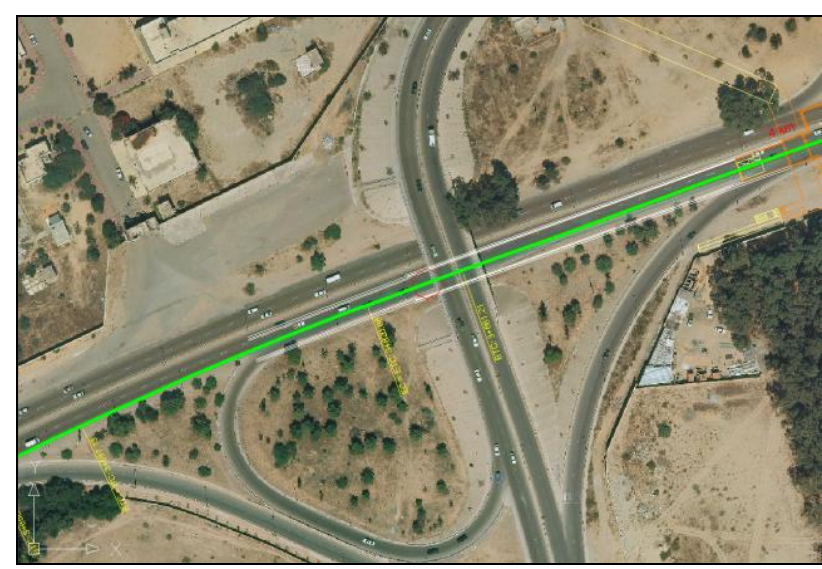

Figure 8. During construction only half of the highway will be closed

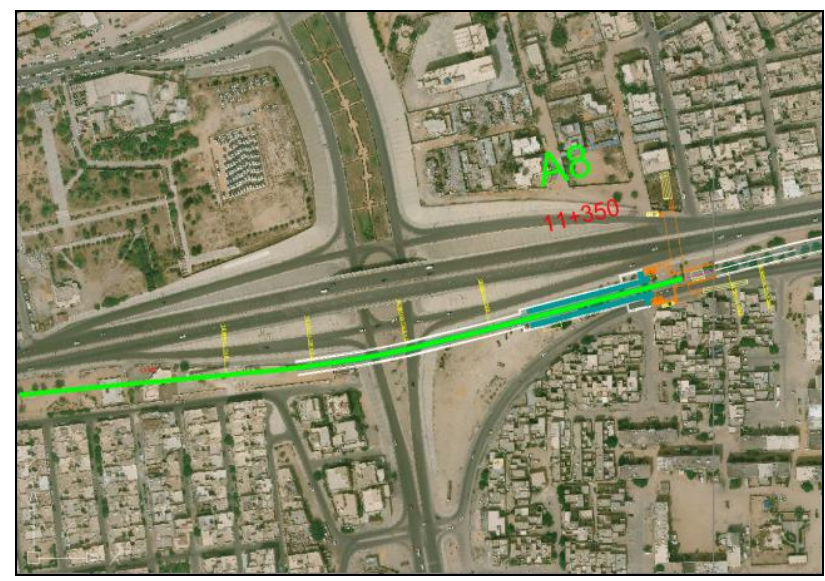

Figure 9. Metro line aligned avoiding a traffic node

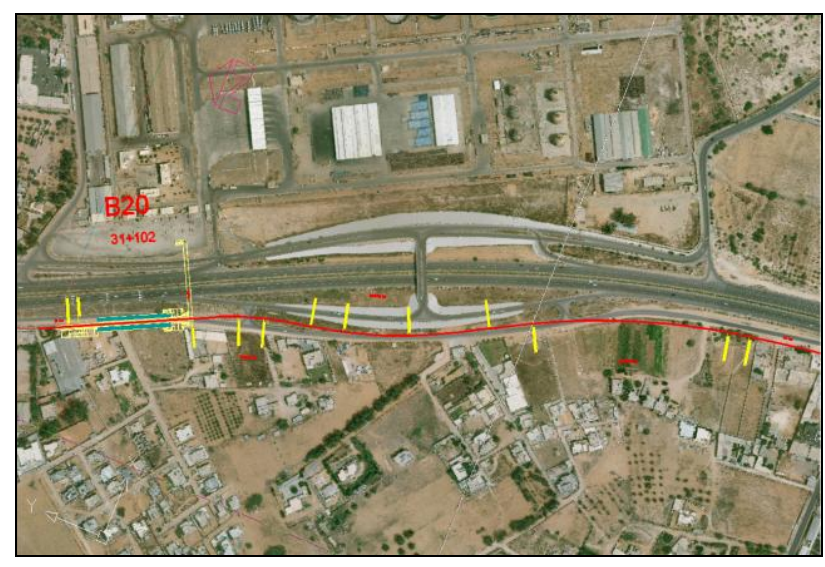

Figure 10. Metro line aligned avoiding bridge foundations

Design criteria taken into consideration during different planning stages were the following:

- The design speed is $80 \mathrm{~km} / \mathrm{h}$.

- Metro lines were designed with minimum curve radius of $550 \mathrm{~m}$ and transient curve of $\mathrm{P}=110 \mathrm{~m}$ (A value).

- During the design work, the designers used values (for the above mentioned) parameters as large as possible.
- During the design work, a maximum attention was paid to the location of stations; the location of stations in essence was determined by the Libyan contrasting partner (RPEMB) in the original (previous) planning study.

- Planning the line alignments was a result of multi-step iterative process:

- In the first step, the stations were placed, and then the line alignment (trail) between stations was planned. In many cases, it was not possible to align the lines with the axis of the stations because of the constraints of the minimal radii and transient curves. Additional constraint meant that the stations might be only imposition on straight line sections.

- In the second step, the line (trail) was drawn and led as close as possible to the problematic station, if any, and station location was slightly modified to have the station fallen on the line and aligned with its direction. This choice was in a way limited in densely built-up areas because of retained buildings. In the case of stations, additional constraining factor was the location of exits; these had to be located in advantageous locations regarding the pedestrian movement patterns.

- The first and second steps were repeated until no compromise or acceptable compromises were reached and the lines and stations imposition into their final locations.

- During planning the alignment lines, special criteria were adopted for these line sections with special characteristics. For example, for the inner city section of line (B) a curve radius of $\mathrm{R}=400 \mathrm{~m}$ was adopted. Another exception was between stations $\mathrm{B} 10$ and $\mathrm{B} 11$, a curve radius of $\mathrm{R}=300 \mathrm{~m}$ was adopted because these two stations were very near to each other, and to avoid the foundations of a planned future high-rise building. In these short sections of the line it presumed that the speed will be decreased.

- During planning, existing and future development in the traffic system were considered, attention was paid to road crossings and nodes, and special caution was paid to the foundations of bridges where the line sections were planned as deep alignment.

- Remotely sensed images and photographs were of great help in planning those sections of the line where subsurface alignment construction was adopted. These sections will be built by cut-and-cover construction. During the construction phase, surface traffic will be insured, and there will be no need for unjustified building demolitions. Surface features, such as roads, buildings, pedestrian pavements, and even young trees and utility poles were easily distinguished and identified in remotely sensed images and aerial photographs, a fact that eased planning the alignment of Metro lines in a way where vehicle and pedestrian traffic is ensured during the construction phase, for example on the sections between B04-B01 and A01-A08 stations (Paulini, 2008).

\section{CONCLUSIONS}

Remotely sensing imagery, whether these were satellite images or aerial photographs, has a spatial accuracy equal or better that $50 \mathrm{~cm}$. Both kinds of images have a high resolution of $60 \mathrm{~cm}$ for satellite images $30 \mathrm{~cm}$ for aerial photographs. Satellite imagery, after carrying out the necessary corrections, transformations, and co-registration has satisfied the required 
accuracy needs in this stage of planning. By producing the necessary orthomaps it was possible to greatly facilitate the field work of the Metro planning teams and to support the in-office work of Metro lines design and imposition of Metro stations in their suitable locations. Nonetheless, the acquired images proved to be cost effective solution during the course of establishing the concept of the traffic study and decision making of the Metro network. At the same time satellite imagery constitutes correct and impressive technical basis for documentation and presentation of plans and technical solutions prepared through the traffic study.

Both the accuracy and resolution are good enough that in the following planning phases the need for terrestrial geodesy will be limited to measuring only required areas. Moreover, the accuracy was sufficient enough to ensure the implementation of the Metro project in later phases.

\section{REFERENCES}

Alhusain, O., 2007. Use of remotely sensed data for urban planning applications, Work report, $80 \mathrm{p}$.

Detrekoi, A., Winkler, G., Alhusain, O., 1996. Environmental monitoring of towns- A Hungarian example, in Schiavoni, U. (ed.) Proceeding of workshop on Information systems and processes for urban civil engineering applications, COST UCE Action C4, EC, Rome, Italy.

Engedy, G., 2008. Maps used in the planning of Tripoli Metro Network, Work report, $57 \mathrm{p}$.

Lillesand, T, Kiefer, R., 1979. Remote sensing and image interpretation, 612 p, John Wiley \& Sons, NY, USA.

Paulini, L., 2008. Planning the Tripoli Metro Network, Work report, $54 \mathrm{p}$. 